\title{
ON THE NUMBER OF DISTINCT EXPONENTS IN THE PRIME FACTORIZATION OF AN INTEGER
}

\author{
CARLO SANNA
}

\begin{abstract}
Let $f(n)$ be the number of distinct exponents in the prime factorization of the natural number $n$. We prove some results about the distribution of $f(n)$. In particular, for any positive integer $k$, we obtain that

$$
\#\{n \leq x: f(n)=k\} \sim A_{k} x
$$

and

$$
\#\{n \leq x: f(n)=\omega(n)-k\} \sim \frac{B x(\log \log x)^{k}}{k ! \log x},
$$

as $x \rightarrow+\infty$, where $\omega(n)$ is the number of prime factors of $n$ and $A_{k}, B>0$ are some explicit constants. The latter asymptotic extends a result of Aktaş and Ram Murty about numbers having mutually distinct exponents in their prime factorization.
\end{abstract}

\section{INTRODUCTION}

Let $n=p_{1}^{a_{1}} \cdots p_{s}^{a_{s}}$ be the factorization of the natural number $n>1$, where $p_{1}<\cdots<p_{s}$ are prime numbers and $a_{1}, \ldots, a_{s}$ are positive integers. Several functions of the exponents $a_{1}, \ldots, a_{s}$ have been studied, including: their product [17], their arithmetic mean [2, 4, 5, 7], and their maximum and minimum $[11,13,15,18]$. See also $[3,8]$ for more general functions.

Let $f$ be the arithmetic function defined by $f(1):=0$ and $f(n):=\#\left\{a_{1}, \ldots, a_{s}\right\}$ for all natural numbers $n>1$. In other words, $f(n)$ is the number of distinct exponents in the prime factorization of $n$. The first values of $f(n)$ are listed in sequence A071625 of OEIS [16].

Our first contribution is a quite precise result about the distribution of $f(n)$.

Theorem 1.1. There exists a sequence of positive real numbers $\left(A_{k}\right)_{k \geq 1}$ such that, given any arithmetic function $\phi$ satisfying $|\phi(k)|<a^{k}$ for some fixed $a>1$, we have that the series

$$
M_{\phi}:=\sum_{k=1}^{\infty} A_{k} \phi(k)
$$

converges and

$$
\sum_{n \leq x} \phi(f(n))=M_{\phi} x+O_{a, \varepsilon}\left(x^{1 / 2+\varepsilon}\right)
$$

for all $x \geq 1$ and $\varepsilon>0$.

From Theorem 1.1 it follows immediately that all the moments of $f$ are finite and that $f$ has a limiting distribution. In particular, we highlight the following corollary:

Corollary 1.1. For each positive integer $k$, we have

$$
\#\{n \leq x: f(n)=k\}=A_{k} x+O_{\varepsilon}\left(x^{1 / 2+\varepsilon}\right),
$$

for all $x \geq 1$ and $\varepsilon>0$.

2010 Mathematics Subject Classification. Primary: 11N25, Secondary: 11N37, 11N64.

Key words and phrases. prime factorization; squarefree numbers; powerful number.

The author is supported by a postdoctoral fellowship of INdAM and is a member of the INdAM group GNSAGA. 
We provides also a formula for $A_{k}$. Before stating it, we need to introduce some notation. Let $\psi$ be the Dedekind function, defined by

$$
\psi(n):=n \prod_{p \mid n}\left(1+\frac{1}{p}\right)
$$

for each positive integer $n$, and let $\left(\rho_{k}\right)_{k \geq 1}$ be the family of arithmetic functions supported on squarefree numbers and satisfying

$$
\rho_{1}(n)=\left\{\begin{array}{ll}
1 & \text { if } n=1, \\
0 & \text { if } n>1,
\end{array} \quad \rho_{k+1}(n)= \begin{cases}0 & \text { if } n=1 \\
\frac{1}{n-1} \sum_{\substack{d \mid n \\
d<n}} \rho_{k}(d) & \text { if } n>1\end{cases}\right.
$$

for all squarefree numbers $n$ and positive integers $k$.

Theorem 1.2. We have

$$
A_{k}=\frac{6}{\pi^{2}} \sum_{n=1}^{\infty} \frac{\rho_{k}(n)}{\psi(n)}
$$

for each positive integer $k$.

Clearly, $f(n) \leq \omega(n)$ for all positive integers $n$, where $\omega(n)$ denotes the number of prime factors of $n$. Motivated by a question of Recamán Santos [14], Aktaş and Ram Murty [1] studied the natural numbers $n$ such that all the exponents in their prime factorization are distinct, that is, $f(n)=\omega(n)$. They called such numbers special numbers (sequence A130091 of OEIS [16]) and they proved the following:

Theorem 1.3. The number of special numbers not exceeding $x$ is

$$
\frac{B x}{\log x}+O\left(\frac{x}{(\log x)^{2}}\right)
$$

for all $x \geq 2$, where

$$
B:=\sum_{\ell} \frac{1}{\ell}
$$

and the sum of over natural numbers $\ell$ that are powerful and special.

Let $g$ be the arithmetic function defined by $g(n):=\omega(n)-f(n)$ for all positive integers $n$. Hence, by the previous observation, $g$ is a nonnegative function and $g(n)=0$ if and only if $n$ is a special number. We prove the following result about $g$, which extends Theorem 1.3 and it is somehow dual to Corollary 1.1.

Theorem 1.4. For each nonnegative integer $k$, we have

$$
\#\{n \leq x: g(n)=k\}=\frac{B x(\log \log x)^{k}}{k ! \log x}\left(1+O_{k}\left(\frac{1}{\log \log x}\right)\right)
$$

for all $x \geq 3$.

Notation. We employ the Landau-Bachmann "Big Oh" notation $O$, as well as the associated Vinogradov symbol «, with their usual meanings. Any dependence of the implied constants is explicitly stated. We reserve the letter $p$ for prime numbers.

\section{Preliminaries}

Recall that a natural number $n$ is called powerful if $p \mid n$ implies $p^{2} \mid n$, for all primes $p$. For all $x \geq 1$, let $\mathcal{P}(x)$ be the set of powerful numbers not exceeding $x$.

Lemma 2.1. We have $\# \mathcal{P}(x) \ll x^{1 / 2}$ for every $x \geq 1$.

Proof. See [9]. 
Lemma 2.2. We have

for all $y \geq 2$.

$$
\sum_{\substack{\ell \in \mathcal{P} \\ \ell>y}} \frac{1}{\ell} \ll \frac{1}{y^{1 / 2}}, \quad \sum_{\ell \in \mathcal{P}(y)} \frac{1}{\ell^{1 / 2}} \ll \log y
$$

Proof. By Lemma 2.1 and by partial summation, we have

$$
\sum_{\substack{\ell \in \mathcal{P} \\ \ell>y}} \frac{1}{\ell}=\left.\frac{\# \mathcal{P}(t)}{t}\right|_{t=y} ^{+\infty}+\int_{y}^{+\infty} \frac{\# \mathcal{P}(t)}{t^{2}} \mathrm{~d} t \ll \int_{y}^{+\infty} \frac{\mathrm{d} t}{t^{1+1 / 2}} \ll \frac{1}{y^{1 / 2}} .
$$

The proof of the second claim is similar.

We need the following upper bound for the number of prime factors of a natural number.

Lemma 2.3. We have

for all integers $n \geq 3$.

$$
\omega(n) \ll \frac{\log n}{\log \log n}
$$

Proof. See, e.g., [6, Proposition 7.10].

For every $x \geq 1$ and every positive integer $h$, let $Q(x ; h)$ denote the number of squarefree numbers not exceeding $x$ and relatively prime with $h$.

Lemma 2.4. We have

$$
Q(x ; h)=\frac{6}{\pi^{2}} \frac{h}{\psi(h)} x+O\left(4^{\omega(h)}\left(x^{1 / 2}+1\right)\right)
$$

for all $x \geq 1$ and all positive integers $h$.

Proof. It follows easily from [10, Eq. 8].

For every $x \geq 1$ and every positive integers $s, h$, let $Q_{s}(x ; h)$ denote the number of squarefree numbers not exceeding $x$, having exactly $s$ prime factors, and relatively prime with $h$.

Lemma 2.5. We have

$$
Q_{s}(x ; h)=\frac{x(\log \log x)^{s-1}}{(s-1) ! \log x}\left(1+O_{\delta, s}\left(\frac{\log \log (h+2)}{\log \log x}\right)\right)
$$

for all $x \geq 3,0<\delta<1$, and for all integers $1 \leq h \leq x^{\delta}$ and $s \geq 1$.

Proof. For $s=1$ the claim follows from the Prime Number Theorem, while for $h=1$ the claim is a classic result of Landau [12]. Hence, suppose $s, h>1$. Also, we can assume $x \geq 3^{1 /(1-\delta)}$. If $n \leq x$ is a squarefree number having exactly $s$ prime factors and such that $(n, h)>1$, then $n=p n^{\prime}$ where $p$ is a prime number dividing $h$ and $n^{\prime} \leq x / p$ is a squarefree number having exactly $s-1$ prime factor. Therefore,

$$
\begin{aligned}
0 & \leq Q_{s}(x ; 1)-Q_{s}(x ; h) \leq \sum_{p \mid h} Q_{s-1}\left(\frac{x}{p}, 1\right) \ll_{s} \sum_{p \mid h} \frac{x}{p} \frac{(\log \log (x / p))^{s-2}}{\log (x / p)} \\
& \ll_{\delta} \frac{x(\log \log x)^{s-2}}{\log x} \sum_{p \mid h} \frac{1}{p} \ll \frac{x(\log \log x)^{s-1}}{\log x} \frac{\log \log (h+2)}{\log \log x},
\end{aligned}
$$

where we used the fact that $p \leq x^{\delta}$ and Mertens' second theorem [6, Theorem 4.5]. Consequently,

$$
\begin{aligned}
Q_{s}(x ; h) & =Q_{s}(x ; 1)+O_{\delta, s}\left(\frac{x(\log \log x)^{s-1}}{\log x} \frac{\log \log (h+2)}{\log \log x}\right) \\
& =\frac{x(\log \log x)^{s-1}}{(s-1) ! \log x}+O_{\delta, s}\left(\frac{x(\log \log x)^{s-1}}{\log x} \frac{\log \log (h+2)}{\log \log x}\right),
\end{aligned}
$$

as claimed. 
Finally, we need a lemma about certain sums of powers.

Lemma 2.6. Let $a_{0}$ be an integer. For all $x_{1}, \ldots, x_{k}>1$ we have

$$
\sum_{a_{0}<a_{1}<\cdots<a_{k}} \frac{1}{x_{1}^{a_{1}} \cdots x_{k}^{a_{k}}}=\frac{1}{\left(x_{1} \cdots x_{k}\right)^{a_{0}}} \prod_{j=1}^{k} \frac{1}{x_{j} \cdots x_{k}-1},
$$

where the sum is over all integers $a_{1}, \ldots, a_{k}$ satisfying $a_{0}<a_{1}<\cdots<a_{k}$.

Proof. We proceed by induction on $k$. For $k=1$, we have

$$
\sum_{a_{0}<a_{1}} \frac{1}{x_{1}^{a_{1}}}=\frac{1}{x_{1}^{a_{0}+1}} \sum_{d=0}^{\infty} \frac{1}{x_{1}^{d}}=\frac{1}{x_{1}^{a_{0}}} \frac{1}{x_{1}-1},
$$

as claimed. Supposing that the claim is true for $k$, we shall prove it for $k+1$. We have

$$
\begin{aligned}
\sum_{a_{0}<\cdots<a_{k+1}} & \frac{1}{x_{1}^{a_{1}} \cdots x_{k+1}^{a_{k+1}}}=\sum_{a_{0}<\cdots<a_{k}} \frac{1}{x_{1}^{a_{1}} \cdots x_{k}^{a_{k}}} \sum_{a_{k}<a_{k+1}} \frac{1}{x_{k+1}^{a_{k+1}}} \\
& =\sum_{a_{0}<\cdots<a_{k+1}} \frac{1}{x_{1}^{a_{1}} \cdots x_{k-1}^{a_{k-1}}\left(x_{k} x_{k+1}\right)^{a_{k}}} \frac{1}{x_{k+1}-1} \\
& =\frac{1}{\left(x_{1} \cdots x_{k+1}\right)^{a_{0}}} \prod_{j=1}^{k} \frac{1}{x_{j} \cdots x_{k+1}-1} \frac{1}{x_{k+1}-1} \\
& =\frac{1}{\left(x_{1} \cdots x_{k+1}\right)^{a_{0}}} \prod_{j=1}^{k+1} \frac{1}{x_{j} \cdots x_{k+1}-1}
\end{aligned}
$$

where we used (2), with $a_{0}$ and $x_{1}$ replaced respectively by $a_{k}$ and $x_{k+1}$, and the induction hypothesis.

\section{Proof of Theorem 1.1}

We begin by proving that for each positive integer $k$ there exists $A_{k}>0$ such that

$$
N_{k}(x):=\#\{n \leq x: f(n)=k\}=A_{k} x+O_{\varepsilon}\left(x^{1 / 2+\varepsilon / 2}\right),
$$

for all $x \geq 1$ and $\varepsilon>0$. Clearly, every natural number $n$ can be written in a unique way as $n=m \ell$, where $m$ is a squarefree number, $\ell$ is a powerful number, and $(m, \ell)=1$. If $m=1$ then $n=\ell$ is powerful and, by Lemma 2.1, belongs to a set of cardinality $O\left(x^{1 / 2}\right)$. If $m>1$ then $f(n)=k$ is equivalent to $f(\ell)=k-1$. Also, for each $\ell$ there are exactly $Q(x / \ell ; \ell)-1$ choices for $m>1$. Therefore, we have

$$
N_{k}(x)=\sum_{\substack{\ell \in \mathcal{P}(x) \\ f(\ell)=k-1}}\left(Q\left(\frac{x}{\ell} ; \ell\right)-1\right)+O\left(x^{1 / 2}\right),
$$

for all $x \geq 1$. For each positive integer $\ell \leq x$, Lemma 2.3 gives $4^{\omega(\ell)} \ll_{\varepsilon} x^{\varepsilon / 4}$. Consequently, by Lemma 2.4, we obtain

$$
Q\left(\frac{x}{\ell} ; \ell\right)=\frac{6}{\pi^{2}} \frac{x}{\psi(\ell)}+O_{\varepsilon}\left(\frac{x^{1 / 2+\varepsilon / 4}}{\ell^{1 / 2}}\right),
$$

for all positive integers $\ell \leq x$. By Lemma 2.2, we have

$$
\sum_{\substack{\ell \in \mathcal{P} \\ \ell>x}} \frac{1}{\psi(\ell)}<\sum_{\substack{\ell \in \mathcal{P} \\ \ell>x}} \frac{1}{\ell} \ll \frac{1}{x^{1 / 2}}
$$


for all $x \geq 1$. In particular, the series

$$
A_{k}:=\frac{6}{\pi^{2}} \sum_{\substack{\ell \in \mathcal{P} \\ f(\ell)=k-1}} \frac{1}{\psi(\ell)}
$$

converges. Also, again by Lemma 2.2, we have

$$
\sum_{\ell \in \mathcal{P}(x)} \frac{1}{\ell^{1 / 2}} \ll \log x \ll_{\varepsilon} x^{\varepsilon / 4} .
$$

At this point, putting together (4) and (5), and using (6) and (8), we obtain

$$
\begin{aligned}
N_{k}(x) & =\sum_{\substack{\ell \in \mathcal{P}(x) \\
f(\ell)=k-1}}\left(\frac{6}{\pi^{2}} \frac{x}{\psi(\ell)}+O_{\varepsilon}\left(\frac{x^{1 / 2+\varepsilon / 4}}{\ell^{1 / 2}}\right)\right)+O\left(x^{1 / 2}\right) \\
& =A_{k} x+O\left(\sum_{\substack{\ell \in \mathcal{P} \\
\ell>x}} \frac{x}{\psi(\ell)}\right)+O_{\varepsilon}\left(\sum_{\ell \in \mathcal{P}(x)} \frac{x^{1 / 2+\varepsilon / 4}}{\ell^{1 / 2}}\right)+O\left(x^{1 / 2}\right) \\
& =A_{k} x+O_{\varepsilon}\left(x^{1 / 2+\varepsilon / 2}\right),
\end{aligned}
$$

as desired. Thus (3) is proved.

Now we shall show that

$$
A_{k} \leq \frac{6}{\pi^{2}} \frac{1}{(k-1) !}
$$

for all positive integers $k$. For $k=1$ the claim is obvious since $A_{1}=6 / \pi^{2}$. Hence, assume $k \geq 2$. If $\ell$ is a powerful number such that $f(\ell)=k-1$, then $\ell=m_{1}^{a_{1}} \cdots m_{k-1}^{a_{k-1}}$ for some integers $m_{1}, \ldots, m_{k-1} \geq 2$ and $2 \leq a_{1}<\cdots<a_{k-1}$. Consequently,

$$
\begin{aligned}
\frac{\pi^{2}}{6} A_{k} & =\sum_{\substack{\ell \in \mathcal{P} \\
f(\ell)=k-1}} \frac{1}{\psi(\ell)}<\sum_{\substack{\ell \in \mathcal{P} \\
f(\ell)=k-1}} \frac{1}{\ell}<\prod_{j=1}^{k-1} \sum_{m=2}^{\infty} \sum_{a=j+1}^{\infty} \frac{1}{m^{a}} \\
& =\prod_{j=1}^{k-1} \sum_{m=2}^{\infty} \frac{1}{m^{j}(m-1)} \leq \prod_{j=1}^{k-1} \frac{1}{j}=\frac{1}{(k-1) !},
\end{aligned}
$$

where we used the facts that

$$
\sum_{m=2}^{\infty} \frac{1}{m(m-1)}=\sum_{m=2}^{\infty}\left(\frac{1}{m-1}-\frac{1}{m}\right)=1
$$

and

$$
\begin{aligned}
\sum_{m=2}^{\infty} \frac{1}{m^{j}(m-1)} & <\frac{1}{2^{j}}+\frac{1}{3^{j} \cdot 2}+\sum_{n=3}^{\infty} \frac{1}{n^{j+1}} \\
& <\frac{1}{2^{j}}+\frac{1}{3^{j} \cdot 2}+\int_{2}^{+\infty} \frac{\mathrm{d} t}{t^{j+1}}=\frac{1}{2^{j}}+\frac{1}{3^{j} \cdot 2}+\frac{1}{j 2^{j}}<\frac{1}{j},
\end{aligned}
$$

for all integers $j \geq 2$. Thus (9) is proved.

Now let $\phi$ be an arithmetic function satisfying $|\phi(k)|<a^{k}$ for all positive integers $k$, where $a>1$ is some constant. From (9) it follows that series (1) converges. Define

$$
y:=2 a+\lfloor C \log x / \log \log (x+2)\rfloor,
$$

where $C>0$ is some absolute constant. Since $f(n) \leq \omega(n)$ for all positive integers $n$, by Lemma 2.3, we can choose $C$ sufficiently large so that $f(n) \leq y$ for all natural numbers $n \leq x$. 
Moreover, from (9) and $y \geq 2 a$, we get that

$$
\sum_{k>y} A_{k} \phi(k) \ll \sum_{k>y} \frac{a^{k}}{(k-1) !}<\frac{a^{y+1}}{y !} \sum_{j=0}^{\infty}\left(\frac{a}{y}\right)^{j} \ll_{a} \frac{a^{y}}{y !} \ll_{a} \frac{1}{x^{1 / 2}},
$$

and

$$
a^{y} y \ll_{a, \varepsilon} x^{\varepsilon / 2},
$$

for all $x \geq 1$. Therefore, putting together (3), (10), and (11), we have

$$
\begin{aligned}
\sum_{n \leq x} \phi(f(n)) & =\sum_{k \leq y} N_{k}(x) \phi(k)=\sum_{k \leq y}\left(A_{k} \phi(k) x+O_{\varepsilon}\left(\phi(k) x^{1 / 2+\varepsilon / 2}\right)\right) \\
& =M_{\phi} x+O\left(\sum_{k>y} A_{k} \phi(k) x\right)+O_{\varepsilon}\left(a^{y} y x^{1 / 2+\varepsilon / 2}\right)=M_{\phi} x+O_{a, \varepsilon}\left(x^{1 / 2+\varepsilon}\right),
\end{aligned}
$$

for all $x \geq 1$ and $\varepsilon>0$. The proof is complete.

\section{Proof of Theorem 1.2}

Recall that $A_{k}$ is defined by (7). For $k=1$ the claim is obvious, since $f(\ell)=0$ if and only if $\ell=1$. Hence, assume $k \geq 2$. If $\ell$ is a powerful number such that $f(\ell)=k-1$, then $\ell$ can be written in a unique way as $\ell=m_{1}^{a_{1}} \cdots m_{k-1}^{a_{k-1}}$, where $1<a_{1}<\cdots<a_{k-1}$ are integers and $m_{1}, \ldots, m_{k-1}>1$ are pairwise coprime squarefree numbers. Therefore, from (7) and Lemma 2.6 we obtain

$$
\begin{aligned}
\frac{\pi^{2}}{6} A_{k} & =\sum_{m_{1}, \ldots, m_{k-1}} \sum_{1<a_{1}<\cdots<a_{k-1}} \frac{1}{\psi\left(m_{1}^{a_{1}} \cdots m_{k-1}^{a_{k-1}}\right)} \\
& =\sum_{m_{1}, \ldots, m_{k-1}} \frac{m_{1} \cdots m_{k-1}}{\psi\left(m_{1} \cdots m_{k-1}\right)} \sum_{1<a_{1}<\cdots<a_{k-1}} \frac{1}{m_{1}^{a_{1}} \cdots m_{k-1}^{a_{k-1}}} \\
& =\sum_{m_{1}, \ldots, m_{k-1}} \frac{1}{\psi\left(m_{1} \cdots m_{k-1}\right)} \prod_{j=1}^{k-1} \frac{1}{m_{j} \cdots m_{k-1}-1},
\end{aligned}
$$

where, here and for the rest of the proof, in summation subscripts $m_{1}, \ldots, m_{k-1}$ are meant to be pairwise coprime, squarefree, and greater than 1. At this point, it is enough to prove that

$$
\sum_{n=m_{1} \cdots m_{k-1}} \prod_{j=1}^{k-1} \frac{1}{m_{j} \cdots m_{k-1}-1}=\rho_{k}(n)
$$

for all squarefree numbers $n>1$. We proceed by induction on $k$. For $k=2$, the claim is true since

$$
\frac{1}{n-1}=\frac{\rho_{1}(1)}{n-1}=\frac{1}{n-1} \sum_{\substack{d \mid n \\ d<n}} \rho_{1}(d)=\rho_{2}(n),
$$

for all squarefree numbers $n>1$. Assuming that the claim is true for $k$, we shall prove it for $k+1$. We have

$$
\begin{gathered}
\sum_{n=m_{1} \cdots m_{k}} \prod_{j=1}^{k} \frac{1}{m_{j} \cdots m_{k}-1}=\frac{1}{n-1} \sum_{m_{1} \mid n} \sum_{n / m_{1}=m_{2} \cdots m_{k}} \prod_{j=2}^{k} \frac{1}{m_{j} \cdots m_{k}-1} \\
=\frac{1}{n-1} \sum_{m_{1} \mid n} \rho_{k}\left(n / m_{1}\right)=\frac{1}{n-1} \sum_{\substack{d \mid n \\
d<n}} \rho_{k}(d)=\rho_{k+1}(n),
\end{gathered}
$$

for all squarefree numbers $n>1$, as desired. The proof is complete. 


\section{Proof of Theorem 1.4}

We have to count the number of positive integers $n \leq x$ such that $g(n)=k$. As in the proof of Theorem 1.1, every $n$ can be written in a unique way as $n=m \ell$, where $m$ is a squarefree number, $\ell$ is a powerful number, and $(m, \ell)=1$. If $m=1$ then $n=\ell$ is powerful and, by Lemma 2.1, belongs to a set of cardinality $O\left(x^{1 / 2}\right)$. If $m>1$ then

$$
\omega(m)=\omega(n)-\omega(\ell)=g(n)+f(n)-f(\ell)-g(\ell)=k+1-g(\ell) .
$$

In particular, $1 \leq \omega(m) \leq k+1$. Assume $x$ sufficiently large, and put $y:=(\log x)^{2}$. Then, by Lemma 2.2, the number of $n \leq x$ such that $\ell>y$ is at most

$$
\sum_{\substack{\ell \in \mathcal{P} \\ \ell>y}} \frac{x}{\ell} \ll \frac{x}{y^{1 / 2}}=\frac{x}{\log x}
$$

Therefore,

$$
M_{k}(x):=\#\{n \leq x: g(n)=k\}=\sum_{s=1}^{k+1} \sum_{\substack{\ell \in \mathcal{P}(y) \\ g(\ell)=k+1-s}} Q_{s}\left(\frac{x}{\ell} ; \ell\right)+O\left(\frac{x}{\log x}\right) .
$$

For each nonnegative integer $r$, put

$$
B_{r}:=\sum_{\substack{\ell \in \mathcal{P} \\ g(\ell)=r}} \frac{1}{\ell}
$$

Note that, in light of Lemma 2.2, the series defining $B_{r}$ converges and, more precisely,

$$
\sum_{\substack{\ell \in \mathcal{P}(y) \\ g(\ell)=r}} \frac{1}{\ell}=B_{r}+O\left(\frac{1}{y^{1 / 2}}\right)=B_{r}+O\left(\frac{1}{\log x}\right) .
$$

Clearly, we can assume $x$ sufficiently large so that $x / y \geq 3$ and $y \leq x^{\delta /(1+\delta)}$, for some fixed $0<\delta<1$. Hence, applying Lemma 2.5 we obtain

$$
\begin{aligned}
Q_{s}\left(\frac{x}{\ell} ; \ell\right) & =\frac{x(\log \log (x / \ell))^{s-1}}{\ell(s-1) ! \log (x / \ell)}\left(1+O_{k}\left(\frac{\log \log (\ell+2)}{\log \log (x / \ell)}\right)\right) \\
& =\frac{x(\log \log x)^{s-1}}{\ell(s-1) ! \log x}\left(1+O_{k}\left(\frac{\log \ell}{\log x}\right)\right)\left(1+O_{k}\left(\frac{\log \log (\ell+2)}{\log \log x}\right)\right) \\
& =\frac{x(\log \log x)^{s-1}}{\ell(s-1) ! \log x}\left(1+O_{k}\left(\frac{\log (\ell+1)}{\log \log x}\right)\right),
\end{aligned}
$$

for all positive integers $s \leq k+1$ and $\ell \leq y$. Consequently,

$$
\begin{aligned}
\sum_{\substack{\ell \in \mathcal{P}(y) \\
g(\ell)=k+1-s}} Q_{s}\left(\frac{x}{\ell} ; \ell\right) & =\frac{x(\log \log x)^{s-1}}{(s-1) ! \log x} \sum_{\substack{\ell \in \mathcal{P}(y) \\
g(\ell)=k+1-s}} \frac{1}{\ell}\left(1+O_{k}\left(\frac{\log (\ell+1)}{\log \log x}\right)\right) \\
& =\frac{x(\log \log x)^{s-1}}{(s-1) ! \log x}\left(B_{k+1-s}+O\left(\frac{1}{\log x}\right)+O_{k}\left(\frac{1}{\log \log x}\right)\right) \\
& =\frac{x(\log \log x)^{s-1}}{(s-1) ! \log x}\left(B_{k+1-s}+O_{k}\left(\frac{1}{\log \log x}\right)\right),
\end{aligned}
$$

where we used (13) and the fact that the series

$$
\sum_{\ell \in \mathcal{P}} \frac{\log (\ell+1)}{\ell}
$$


converges. Thus, putting together (12) and (14), and noting that $B_{0}=B$, we obtain

$$
M_{k}(x)=\frac{B x(\log \log x)^{k}}{k ! \log x}\left(1+O_{k}\left(\frac{1}{\log \log x}\right)\right),
$$

as desired. The proof is complete.

\section{REFERENCES}

1. K. Aktaş and M. Ram Murty, On the number of special numbers, Proc. Indian Acad. Sci. Math. Sci. 127 (2017), no. 3, 423-430.

2. Hui Zhong Cao, On the average of exponents, Northeast. Math. J. 10 (1994), no. 3, 291-296.

3. - Functions involving the number of prime factors of a natural number, Acta Math. Sinica (Chin. Ser.) 39 (1996), no. 5, 602-608.

4. J.-M. De Koninck, Sums of quotients of additive functions, Proc. Amer. Math. Soc. 44 (1974), 35-38.

5. J.-M. De Koninck and A. Ivić, Sums of reciprocals of certain additive functions, Manuscripta Math. 30 (1979/80), no. 4, 329-341.

6. J.-M. De Koninck and F. Luca, Analytic number theory, Graduate Studies in Mathematics, vol. 134, American Mathematical Society, Providence, RI, 2012, Exploring the anatomy of integers.

7. R. L. Duncan, On the factorization of integers, Proc. Amer. Math. Soc. 25 (1970), 191-192.

8. __ Some applications of the Turán-Kubilius inequality, Proc. Amer. Math. Soc. 30 (1971), 69-72.

9. S. W. Golomb, Powerful numbers, Amer. Math. Monthly 77 (1970), 848-855.

10. D. G. Hazlewood, On k-free integers with small prime factors, Proc. Amer. Math. Soc. 52 (1975), 40-44.

11. I. Kátai and M. V. Subbarao, On the maximal and minimal exponent of the prime power divisors of integers, Publ. Math. Debrecen 68 (2006), no. 3-4, 477-488.

12. E. Landau, Sur quelques problèmes relatifs à la distribution des nombres premiers, Bull. Soc. Math. France 28 (1900), 25-38.

13. I. Niven, Averages of exponents in factoring integers, Proc. Amer. Math. Soc. 22 (1969), 356-360.

14. B. Recamán Santos, Consecutive numbers with mutually distinct exponents in their canonical prime factorization, http://mathoverflow. net/questions/201489.

15. K. Sinha, Average orders of certain arithmetical functions, J. Ramanujan Math. Soc. 21 (2006), no. 3, 267-277.

16. N. J. A. Sloane, The On-Line Encyclopedia of Integer Sequences, http://oeis.org.

17. D. Suryanarayana and R. Sitaramachandra Rao, The number of square-full divisors of an integer, Proc. Amer. Math. Soc. 34 (1972), 79-80.

18. - On the maximum and minimum exponents in factoring integers, Arch. Math. (Basel) 28 (1977), no. 3, 261-269.

Università degli Studi di Genova, Department of Mathematics, Genova, Italy

E-mail address: carlo.sanna.dev@gmail.com 